\title{
Structural changes in the early stages of Duchenne muscular dystrophy
}

\author{
W. G. BRADlEY, P. HUDGSON, P. F. LARSON, T. A. PAPAPETROPOULOS, \\ AND M. JENKISON
}

From the Regional Neurological Centre, Newcastle General Hospital, Newcastle upon Tyne

SUMMARY The finding of a relative absence of degeneration and regeneration in a muscle biopsy taken at $2 \frac{1}{2}$ weeks of age from a boy who later showed the florid pathological changes of preclinical Duchenne muscular dystrophy prompted a review of muscle biopsies taken from boys in the preclinical and early clinical stages of this disease. Only one other biopsy obtained in the first year of life was available. In this, taken at 2 months, there were active changes present. These findings suggest that biopsies taken to detect preclinical cases should not be performed at less than 2 months of age, and raise the possibility of the existence of a stage in Duchenne muscular dystrophy before active degeneration when the muscle shows little or no change on light microscopy.

The pathological changes in skeletal muscle and the changes in the serum levels of enzymes released from muscle in established cases of Duchenne muscular dystrophy are well-known. In the past 10 years it has been recognized that at a time (termed the preclinical stage) before the boys develop clinical signs of this disease, the serum levels of these enzymes are raised, the peak being at about the age of 18 months with a progressive fall thereafter (Pearson, 1957; Pearce, Pennington, and Walton, 1964; Heyck, Laudahn, and Carsten, 1966). In the preclinical stage muscle necrosis, phagocytosis, and regeneration appear to be far more active than in the later clinical stage (Pearson, 1962; Hudgson, Pearce, and Walton, 1967).

Investigation during the first few weeks of life of a boy at risk for Duchenne muscular dystrophy is sometimes required for prognostication, particularly before adoption of the child, and for genetic counselling. In such a boy, who later developed clinical signs of the disease and florid changes in the muscle biopsy, we found that a muscle biopsy taken at the age of $2 \frac{1}{2}$ weeks showed none of the active necrosis and phagocytosis typical of the preclinical stage. In view of this finding, we have reviewed our muscle biopsies taken from boys under the age of 7 years, who either showed signs of Duchenne muscular dystrophy, or who were in the preclinical stage at the time of biopsy.

\section{CASE HISTORY}

The patient, G.P., was born after a full-term normal pregnancy, and appeared normal at birth. His mother, two brothers of whom had Duchenne muscular dystrophy, had a normal serum creatine kinase activity (25 i.u.) and a normal electromyogram (Dr. David Gardner-Medwin). The serum creatine kinase activity on cord blood was 1,600 i.u., and the child was investigated at the age of $2 \frac{1}{2}$ weeks. The serum creatine kinase activity at that time was 1,433 i.u., and a biopsy of the left quadriceps muscle showed abnormal variability of fibre size, a moderate number of hyaline fibres, and a slight increase of fibrous connective tissue. There was no certain phagocytosis or regeneration (Figs 1 and 2). Because these changes differed strikingly from previous experience with preclinical muscular dystrophy, the diagnosis at that time was uncertain. At the age of 2 years 4 months the serum creatine kinase activity was 7,400 i.u., and at the age of 3 years 2 months, when he was still clinically normal, the creatine kinase was 2,160 i.u. A biopsy of the right quadriceps muscle at this time showed the changes typically reported in the preclinical stage (Figs 3 and 4).

\section{METHODS}

Muscle biopsies taken from 19 boys with Duchenne muscular dystrophy at the age of 6 years or less were reviewed. Several of these boys had minimal signs of the disease, and were investigated early because of a family history of the disease. In addition, 


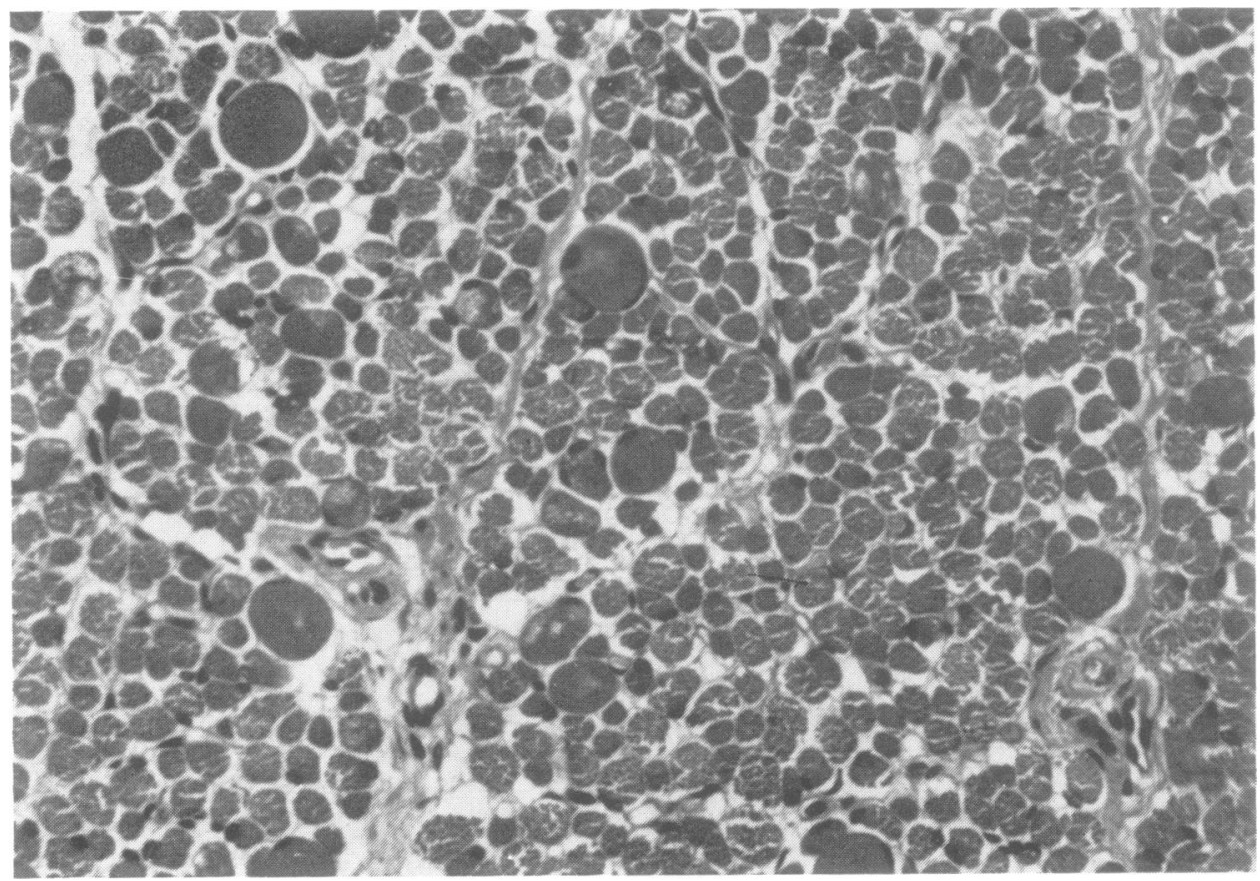

FIG. 1. G.P. Quadriceps biopsy age $2 \frac{1}{2}$ weeks. Transverse section showing an abnormal number of hyaline fibres, a slightly increased variability of fibre size, and rounding of fibres with increased endomysial fibrous tissue. $H$ and $E, \times 384$.

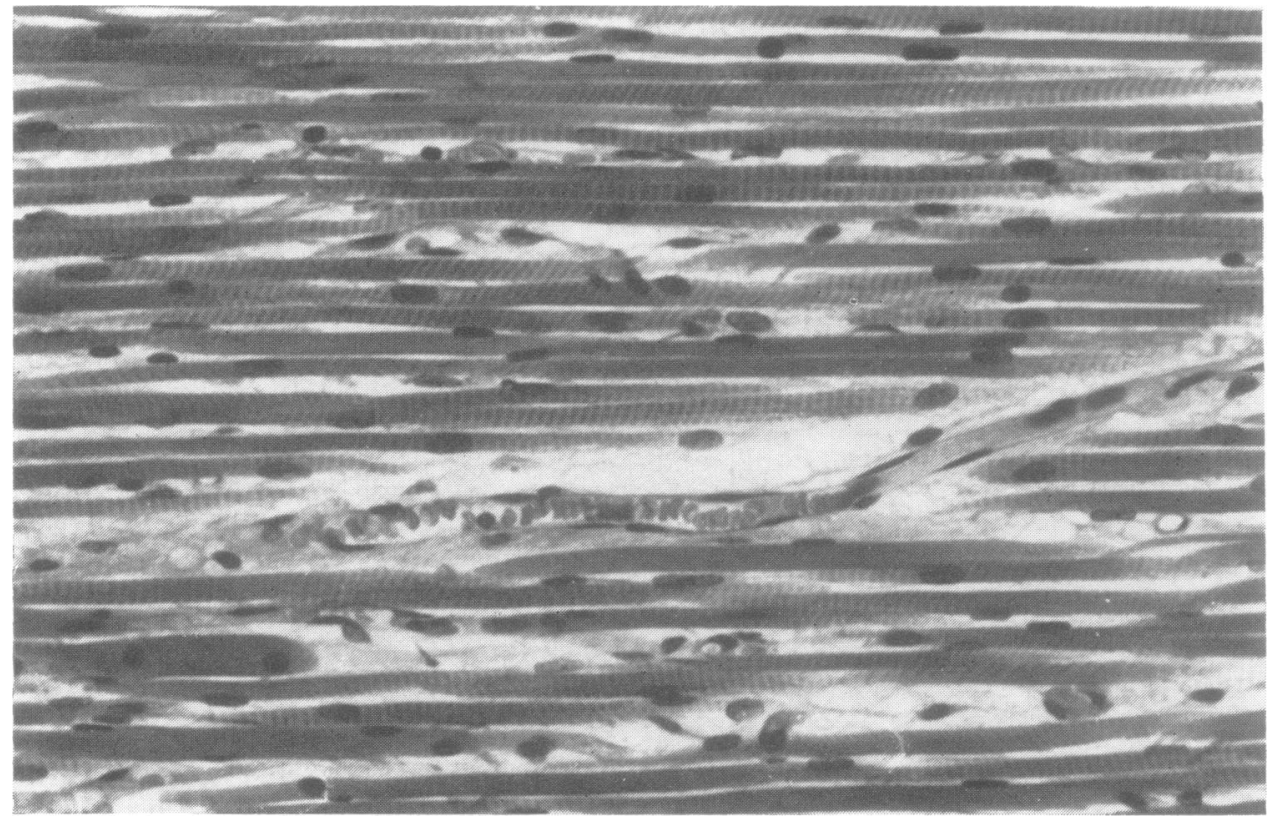

FIG. 2. G.P. As Fig. 1, longitudinal section. $H$ and $E, \times 384$. 


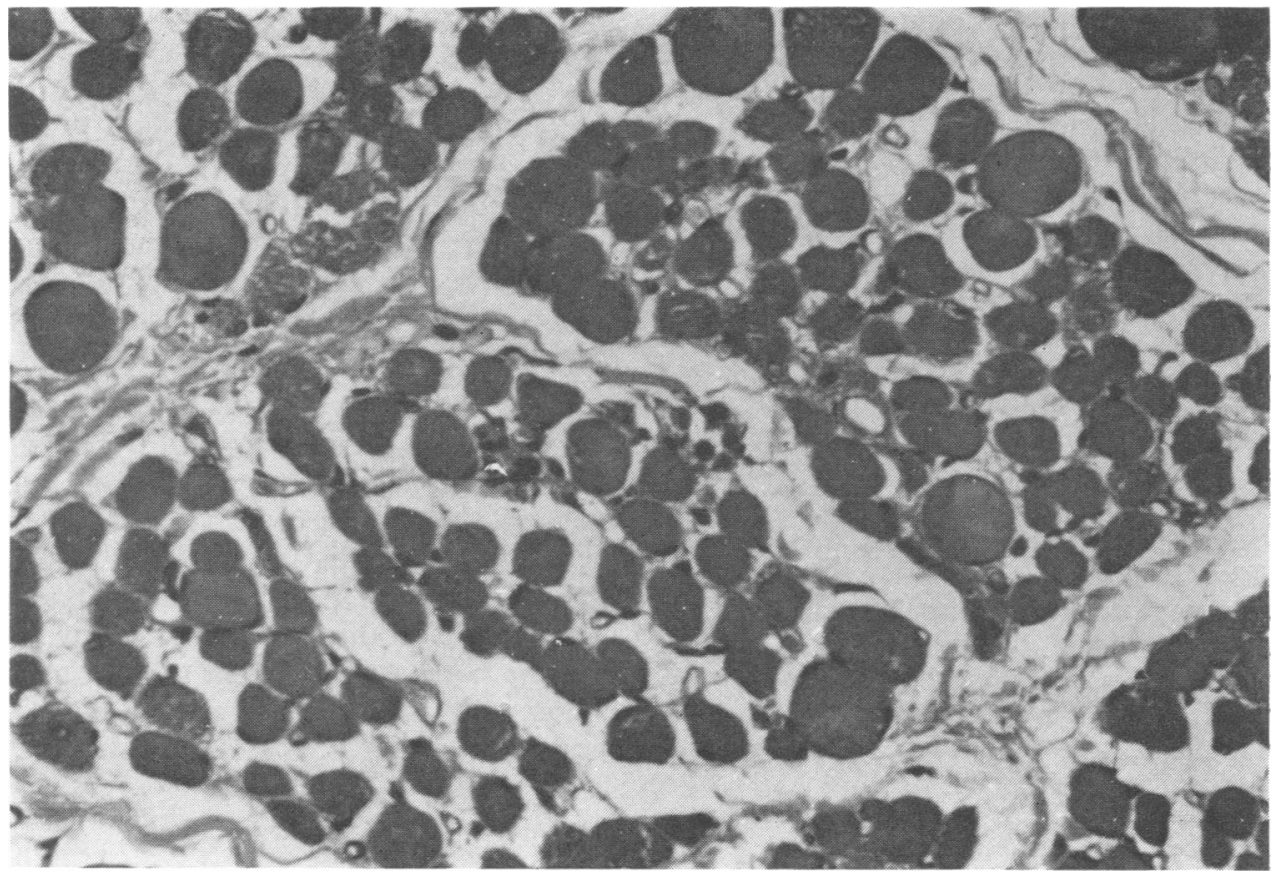

FIG. 3. G.P. Quadriceps muscle biopsy age 3 years 2 months. Transverse section showing hyaline, necrotic, and regenerating fibres, with increased endomysial fibrosis. $H$ and $E, \times 384$.

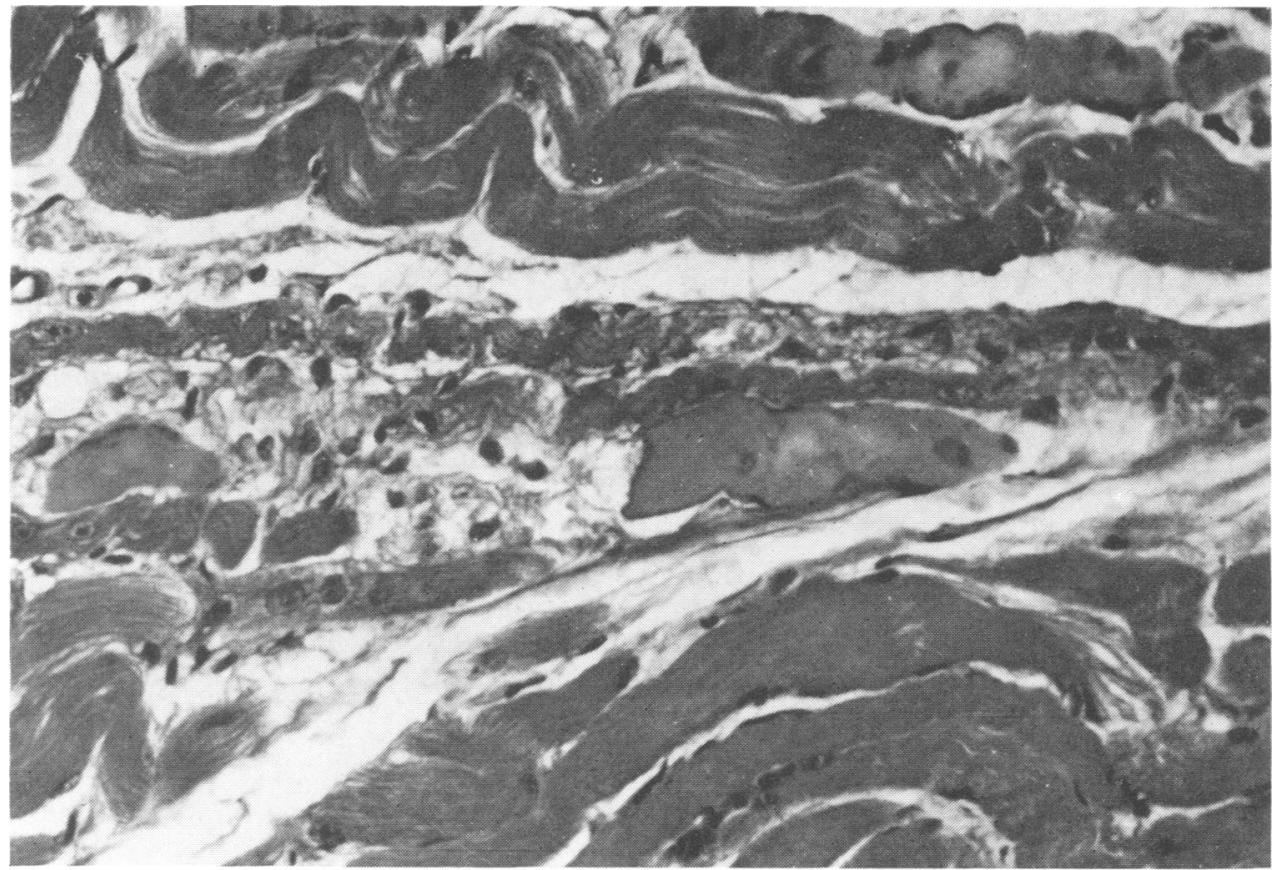

FIG. 4. G.P. As Fig. 3, longitudinal section showing necrotic fibres undergoing phagocytosis, and chains of nuclei in regenerating fibres. $H$ and $E, \times 384$. 
biopsies obtained from three boys in the preclinical stage were reviewed. Twenty biopsies of limb muscles from 19 patients were available. Sixteen biopsies were from the quadriceps femoris muscle, and the remainder from biceps, triceps, gastrocnemius, and deltoid muscles. The diagnosis in these cases was based on typical clinical findings of the distribution of muscle weakness and wasting, the age of onset, and the rate of progression of the disease. There was a family history of affected cases in two generations in four, of affected uncles in three, and of affected siblings in four; eight cases were sporadic. The serum creatine kinase activity was estimated in 17 and found to lie in the range of 100 to 400 times normal. In six the electromyogram was myopathic. Three cases have previously been reported by Hudgson et al. (1967).

The light microscopic abnormalities of abnormal variation in fibre size, the proportion of hyaline fibres, phagocytosis, regeneration, and fibrosis were each graded, and the average taken of the various observers' scores.

\section{RESULTS}

The only biopsy we have from patients less than 2 months of age was that from G.P. described above. Biopsies from patients in the preclinical stage aged 2 months, 1 year, and 2 years showed active necrosis, phagocytosis, and regeneration, which have previously been reported as typical of the preclinical stage of Duchenne muscular dystrophy.

Figure 5 shows the grading of histological $\stackrel{\vec{F}}{\stackrel{\vec{S}}{+}}$ abnormalities in the 20 biopsies. Though there? is a considerable variation between individual $\frac{\text { 을 }}{5}$ biopsies, the tendency with increasing age is for $\frac{\text { ? }}{\overrightarrow{0}}$ a gradual increase in the amount of variability of 0 fibre size and of phagocytosis, but a gradual o decrease in the amount of regenerative activity. $\vec{O}$

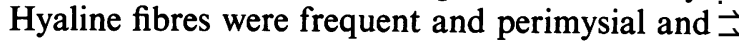
endomysial fibrosis prominent even in the ${ }^{\omega}$ biopsies from the youngest patients. The degree

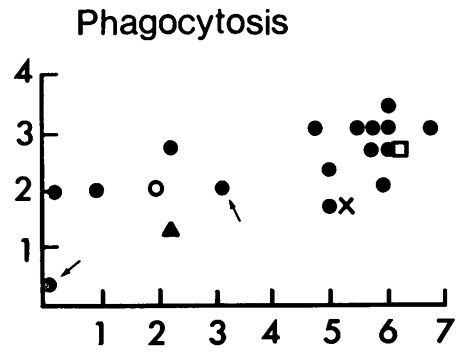

Variation in fibre size

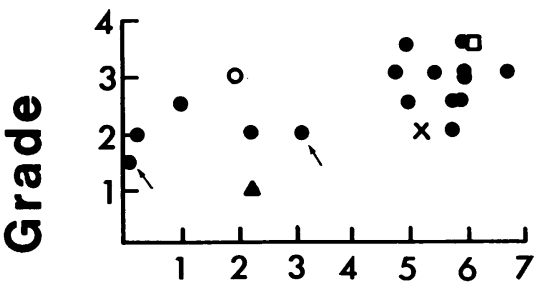

Fibrosis

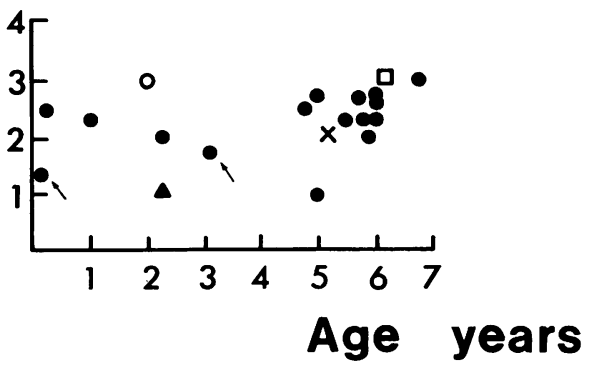

Regeneration

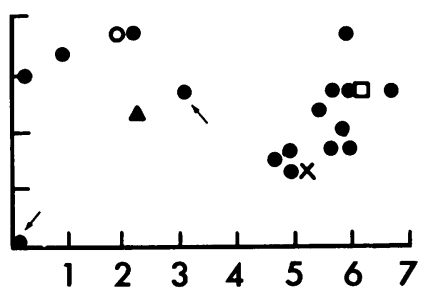

Hyaline fibres

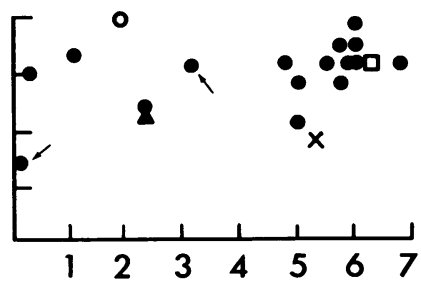

FIG. 5. Graphs showing the change with age in the degree $\stackrel{\varnothing}{\varnothing}$ of phagocytosis of fibres, $\vec{\overrightarrow{ }}$ regeneration, variation in 3 fibre size, fibrosis, and the frequency of hyaline fibres? seen in the 20 muscle biopsies. (For details of grad-ב ing see Methods section.)
- QUADS

$\times$ BICEPS

- GASTROC

- TRICEPS

- DELTOID

Patient G.P. 
of these latter abnormalities remained approximately constant up till the age of 6 years.

\section{DISCUSSION}

This study was prompted by the finding of only minor changes in a muscle biopsy taken at the age of $2 \frac{1}{2}$ weeks from a boy, who at the age of 3 years had florid changes characteristic of the preclinical stage of Duchenne muscular dystrophy in a repeat muscle biopsy. Only one other biopsy within the first year of life was available for review, taken at the age of 2 months (previously reported by Hudgson et al., 1967), which showed active necrosis, phagocytosis, and regeneration. From the practical point of view these findings suggest that it is important to delay the biopsy of the muscle until at least the age of 2 months in patients who require to be investigated soon after birth.

The earliest stages of Duchenne muscular dystrophy have yet to be explored. Whether the muscle is at any time structurally and functionally normal is unknown. While studies of muscle from affected male foetuses obtained by therapeutic abortion of known carriers of the disease could provide this information, the identification of these foetuses has proved difficult. Since the serum creatine kinase activity in the umbilical cord blood of 36 foetuses therapeutically aborted for psychiatric and sociological reasons (and assumed to be normal) was up to 995 i.u., the serum creatine kinase activity in the dystrophic foetus would have to be greatly elevated for identification (Larson and Park, 1972). Larson and Jenkison (1972) found myotubes in the quadriceps muscle of a 24 week male foetus aborted from a known carrier of Duchenne muscular dystrophy. Myotubes had already disappeared from normal foetuses by that stage of gestation. While these findings suggested a delay in maturation of skeletal muscle in that foetus, the identification and study of more affected foetuses is required to show the sequence of events in myogenesis in this disease.

The presence of hyaline fibres and endomysial fibrosis in biopsies at the earliest times after delivery in this disease suggests that these changes are not secondary to muscle fibre necrosis, phagocytosis, and regeneration, but rather a primary response. The degree of these abnormalities did not change significantly in the first six years of life. On the other hand, this review of the muscle from the preclinical and early clinical stages of Duchenne muscular dystrophy confirms earlier experience that there is an increasing amount of variation in fibre size and phagocytosis, and a decreasing amount of regeneration with increasing age.

We thank Professor John Walton for advice and for reading the manuscript. Dr. R. J. T. Pennington and Dr. D. Park kindly measured the serum creatine kinase activities. We are grateful to our gynaecological colleagues for allowing access to the products of therapeutic abortions. This study was performed with the aid of grants from the Muscular Dystrophy Group of Great Britain, the Muscular Dystrophy Associations of America, Inc. and the Medical Research Council. W.G.B. was holder of a Wellcome Senior Research Fellowship in Clinical Science. P.F.L. was supported by a George Meany Postdoctoral Fellowship from the Muscular Dystrophy Associations of America, Inc.

\section{REFERENCES}

Heyck, H., Laudahn, G. and Carsten, P.-M. (1966). Enzymaktivitätsbestimungen bei Dystrophia musculoren progressiva. IV. Mitteilung. Die Serumenzymkinetik im präklinischen Stadium des Typus Duchenne während der ersten2 Lebensjahre. Klinische Wochenschrift, 44, 695-700.

Hudgson, P., Pearce, G. W. and Walton, J. N. (1967). Preclinical muscular dystrophy: histopathological changes observed on muscle biopsy. Brain, 90, 565-576.

Larson, P. F., and Jenkison, M. (1972). (In preparation.)

Larson, P. F., and Park, D. (1972). (In preparation.)

Pearce, J. M. S., Pennington, R. J. and Walton, J. N. (1964). Serum enzyme studies in muscle disease. Part II. Serum creatine kinase activity in muscular dystrophy and in other myopathic and neuropathic disorders. Journal of Neurology, Neurosurgery, and Psychiatry, 27, 96-99.

Pearson, C. M. (1957). Serum enzymes in muscular dystrophy and certain other muscular and neuromuscular diseases. I. Serum glutamic oxalacetic transaminase. New England Journal of Medicine, 256, 1069-1075.

Pearson, C. M. (1962). Histopathological features of muscle in the preclinical stage of muscular dystrophy. Brain, 85 , $109-120$. 